\title{
Development of a Laser-Assisted Chemical Vapor Deposition System for the Growth of Carbon Nanotubes
}

\author{
Takashi Uchida ${ }^{* 1,{ }^{*}}$ and Yoshikazu Yoshida ${ }^{* 1,{ }^{*} 2}$ \\ ${ }^{* 1}$ Faculty of Science and Engineering, Toyo University, 2100, Kujirai, Kawagoe, Saitama, Japan, \\ $350-8585$ \\ E-mail: uchida_t@toyo.jp \\ ${ }^{* 2}$ Bio-Nano Electronics Research Centre, Toyo University, 2100, Kujirai, Kawagoe, Saitama, Japan, \\ 350-8585
}

\begin{abstract}
We developed the chemical vapor deposition (CVD) system in which the pulsed UV laser (a fourth-harmonic Nd:YAG laser, $\lambda=266 \mathrm{~nm}$, pulse width $=4.3 \mathrm{~ns}$, repetition frequency $=10 \mathrm{~Hz}$ ) can be introduced. This CVD system is based on the cold-wall thermal CVD. The substrate, on which the growth catalysts are deposited, can be heated by resistance heating and be irradiated with UV laser. We used a commonly-used Fe/Co supported on zeolite catalyst for carbon nanotube growth. The catalyst was coated on a $10 \mathrm{~mm} \times 10 \mathrm{~mm}$ Si substrate. Ethanol gas was used as a carbon source. In the CVD process, the substrate is heated by resistance heating at $300{ }^{\circ} \mathrm{C}$, then ethanol gas is supplied in the CVD chamber with the pressure around $1000 \mathrm{~Pa}$, after that the laser is irradiated to the substrate. We have examined the dependence of the laser fluence on the grown carbon nanotubes (CNTs). The growth area and growth amount of CNTs depend on the laser fluence. CNTs were synthesized at the laser spot with the laser fluence of $0.05 \mathrm{~J} / \mathrm{cm}^{2}$.

DOI:10.2961/jlmn.2011.03.0008
\end{abstract}

Keywords: Laser-assisted CVD, UV laser, carbon nanotubes, scanning electron microscope, Raman spectroscopy, $266 \mathrm{~nm}$

\section{Introduction}

Carbon nanotubes (CNTs) are the promising building blocks for nanotechnology [1]. One of the greatest features of CNTs is that the electronic states of them can be both semiconducting and metallic depending on their chirality [2]. Therefore, CNTs are expected to be applied as a wiring material, a field effect transistor and so on. In order to realize such applications, growth techniques should be studied.

Generally, CNTs are synthesized by several methods, such as arc discharge, laser vaporization, and chemical vapor deposition (CVD). Among these synthesis methods, CVD is thought to be suitable to realize above-mentioned practical applications, because the number of growth parameters for CVD is larger than the other synthesis methods. However, such practical applications have not been yielded yet. The precise control of the growth position and the lowering of the growth temperature are necessary.

The laser-assisted CVD (LACVD) has been used to study the growth of CNTs [3-12]. In the LACVD, the laser is irradiated to the substrate on which the metallic catalysts are deposited. The role of the laser irradiation is to heat the substrate locally. Therefore, visible or infrared lights were used in the early studies. One can get CNTs only on the substrate where the laser is irradiated. And using the LACVD, the heating duration is precisely controllable. Thus, the LACVD growth of CNTs has been investigated in order to control the growth position and also to clarify the growth mechanism of CNTs. In the earlier studies, a cw laser or a millisecond pulse laser were used. However, the heating duration, which relates to the laser irradiation time, is too long to control the growth of CNTs and to analyze the growth process of CNTs precisely. We expect that the UV light, which can dissociate the carbon feedstock, is effective to reduce the substrate heating temperature for the CNT growth and that the shorter pulse width is effective to observe the growth process of the CNTs precisely.

In this study, we developed the CVD system in which the pulsed UV laser can be introduced. And we synthesized single-walled CNTs (SWCNTs) using a commonly-used $\mathrm{Fe} / \mathrm{Co}$ supported on zeolite catalyst.

\section{Experimental}

\subsection{Developed CVD apparatus}

Fig. 1(a) shows the setup of the laser-irradiated CVD apparatus. This apparatus is mainly composed of a laser, an optical system, and a CVD chamber. A fourth-harmonic Nd:YAG laser (Quantel, Brilliant, $\lambda=266 \mathrm{~nm}$, pulse width $=4.3 \mathrm{~ns}$, repetition frequency $=10 \mathrm{~Hz}$ ) is used for the irradiation. The laser light is focused by using a single planeconvex lens ( $\mathrm{f}=75 \mathrm{~mm} @ 546.1 \mathrm{~nm})$. The CVD chamber is installed on a xyz stage. We can control the irradiation position on the substrate by moving the CVD chamber using the xyz stage.

Fig. 1(b) shows the detail of the CVD chamber. This is composed of a vacuum chamber, a vacuum pump, and a feedstock. This CVD chamber is based on the cold-wall thermal CVD system. A six-way cube with DN40 conflat flanges were used as a vacuum chamber. A quartz viewport was connected to the top flange of the chamber. The vacuum pump, the feedstock, and the electric feedthrough were connected to the side flanges. Other two flanges were blanked. The growth substrate is connected to the electric 
feedthrough in order to heat the substrate by resistance heating and also to hold the substrate in the vacuum chamber. In order to minimize the heating loss, we installed the tantalum foil between the substrate and the electrodes of the feedthrough. The substrate temperature was monitored by using a thermocouple, which is directly attached to the substrate. We used a complete dry turbo molecular pump station (Pfeiffer-Vacuum, Hi Cube 80 Eco). The base pressure of the vacuum chamber is below $5 \times 10^{-3} \mathrm{~Pa}$.

\subsection{Growth of CNTs}

An n-type low-resistance Si substrate $[10 \mathrm{~mm} \times 10 \mathrm{~mm}$ Si (100), resistivity $\leq 10 \Omega \mathrm{cm}]$ was used for the synthesis. A commonly-used $\mathrm{Co} / \mathrm{Fe}$ supported on the $\mathrm{y}$-type zeolite powder was used as a catalyst.

This metal catalyst was prepared by dissolving $2.5 \mathrm{wt} \%$ Co acetate and $2.5 \mathrm{wt} \% \mathrm{Fe}$ acetate in $40 \mathrm{ml}$ of ethanol and then mixing them with the y-type zeolite by ultrasonication. The mixture solution was then uniformly deposited on the Si substrate by the process of spin-coating. Ethanol vapor was used as a carbon source.

In the CVD process, the substrate was heated by resistance heating at $300{ }^{\circ} \mathrm{C}$ or $500{ }^{\circ} \mathrm{C}$, then ethanol gas was supplied in the chamber with the pressure around $1000 \mathrm{~Pa}$, after that the laser was irradiated to the substrate. We have examined the effect of the laser irradiation, and the dependence of the irradiated laser fluence on the grown CNTs. The CVD conditions are shown in Table 1.

\subsection{Characterization}

The synthesized CNTs were characterized by scanning electron microscopy (SEM, JEOL, JSM-7400F) and microRaman spectroscopy (Horiba Jobin Ybon, HR800UV) with excitation wavelengths of $633 \mathrm{~nm}$.

\section{Results and discussion}

\subsection{CNT growth without laser irradiation}

In order to check the operation of the developed CVD apparatus, we performed the CVD without laser irradiation. We examined the two substrate temperatures $T_{\text {sub }}=500{ }^{\circ} \mathrm{C}$

(a)
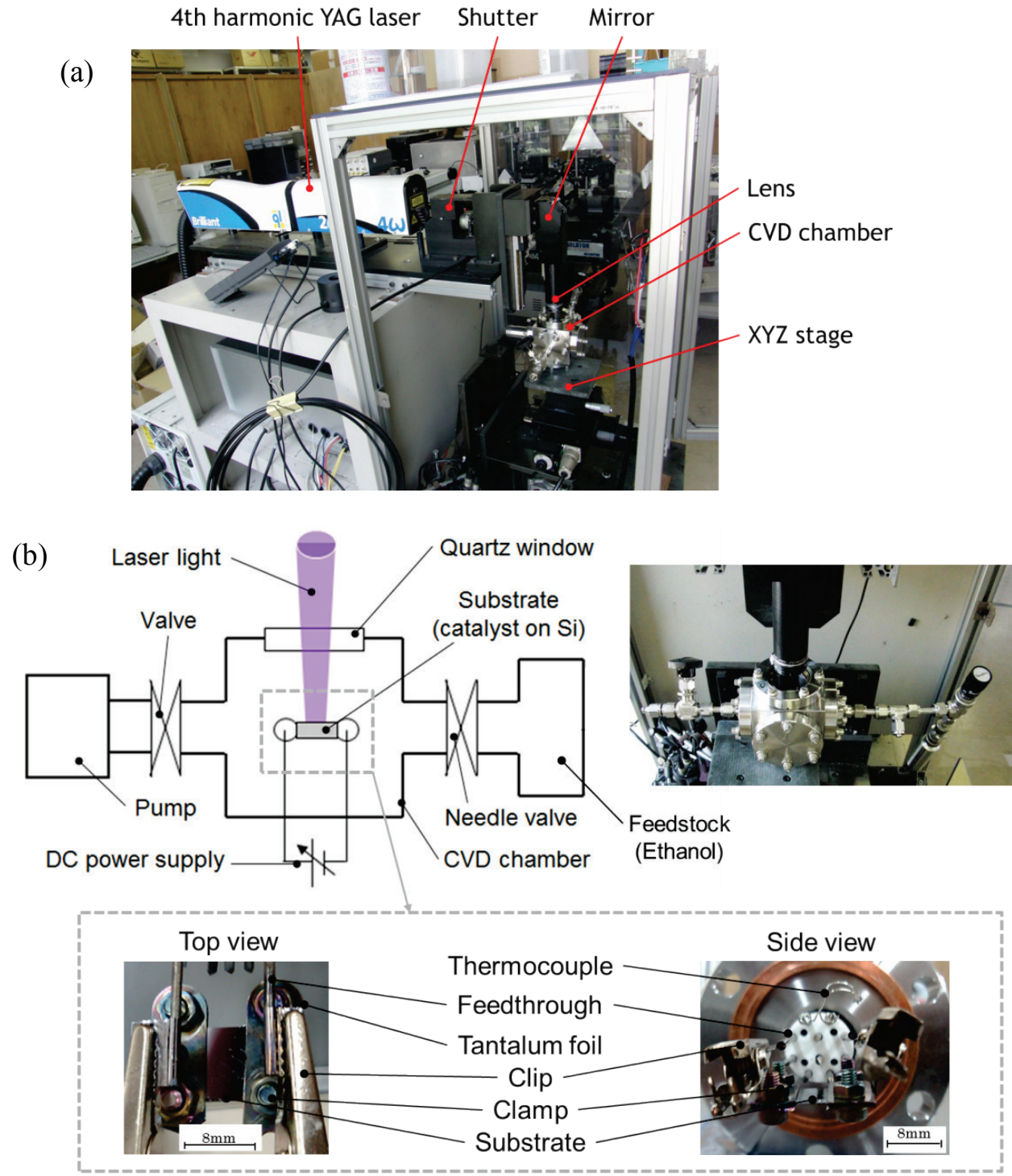

Fig. 1 (a) Setup of the laser-assisted CVD apparatus (b) detail of the CVD chamber 
Table 1 CVD conditions, where $\lambda, W_{\text {pulse, }} f_{\text {rep }}, D_{\text {spot }}, F, T_{\text {sub }}, P_{\mathrm{CVD}}$, and $D_{\mathrm{CVD}}$, are the wavelength, pulse width, repetition frequency, laser spot diameter, laser fluence per pulse, substrate temperature, chamber pressure during CVD, and growth duration, respectively.

\begin{tabular}{|c|c|c|c|c|c|c|c|c|c|}
\hline \multirow[b]{2}{*}{ No. } & \multicolumn{5}{|c|}{ Laser irradiation (LI) } & \multirow{2}{*}{$\begin{array}{l}T_{\text {sub }} \\
\left({ }^{\circ} \mathrm{C}\right)\end{array}$} & \multirow{2}{*}{$\begin{array}{c}P_{\mathrm{CVD}} \\
(\mathrm{Pa})\end{array}$} & \multirow{2}{*}{$\begin{array}{l}D_{\mathrm{CVD}} \\
(\mathrm{sec})\end{array}$} & \multirow[b]{2}{*}{ Note } \\
\hline & $\begin{array}{c}\lambda \\
(\mathrm{nm})\end{array}$ & $\begin{array}{l}W_{\text {pulse }} \\
\text { (ns) }\end{array}$ & $\begin{array}{c}f_{\text {rep }} \\
(\mathrm{Hz})\end{array}$ & $\begin{array}{c}D_{\text {spot }} \\
(\mathrm{mm} \phi)\end{array}$ & $\begin{array}{c}F \\
\left(\mathrm{~J} / \mathrm{cm}^{2}\right)\end{array}$ & & & & \\
\hline 1 & - & - & - & - & - & 500 & 1000 & 600 & higher $T_{\text {sub }}$, without LI \\
\hline 2 & - & - & - & - & - & 300 & 1000 & 600 & lower $T_{\text {sub }}$, without LI \\
\hline 3 & 266 & 4.3 & 10 & 0.33 & 3.3 & 300 & 1000 & 100 & lower $T_{\text {sub }}$, highest $F$ \\
\hline 4 & 266 & 4.3 & 10 & 0.33 & 2.2 & 300 & 1000 & 100 & lower $T_{\text {sub }}$, medium $F$ \\
\hline 5 & 266 & 4.3 & 10 & 0.33 & 1.1 & 300 & 1000 & 100 & lower $T_{\text {sub }}$, medium $F$ \\
\hline 6 & 266 & 4.3 & 10 & 5 & 0.05 & 300 & 1000 & 100 & lower $T_{\text {sub }}$, lowest $F$ \\
\hline
\end{tabular}
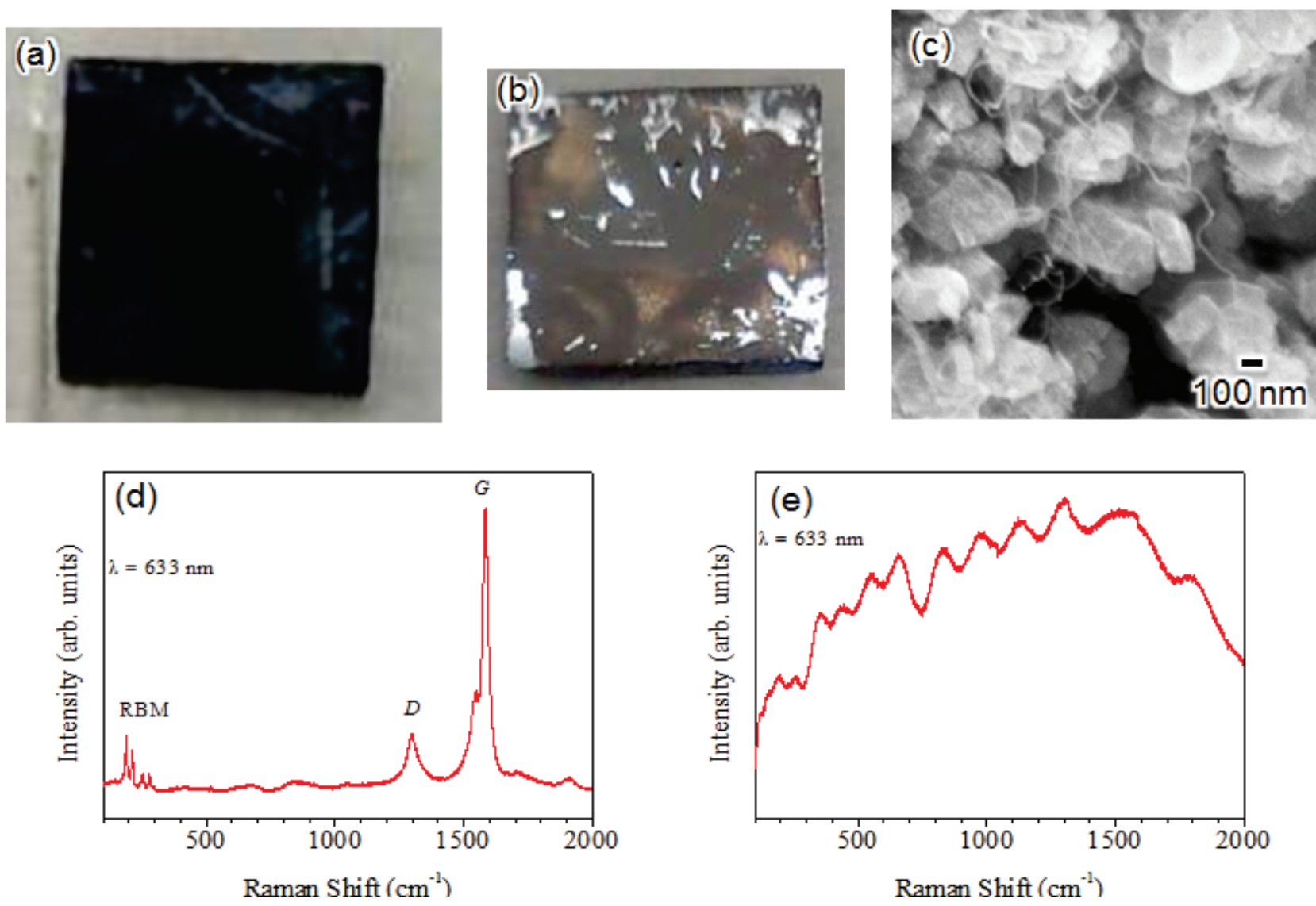

Fig. 2 (a), and (b) Photos of the substrates after CVD with the condition No. 1 and No. 2, respectively. (c) SEM image of the substrates after CVD with the condition No. 1. (d), and (e) Raman spectra of the substrates after CVD with the condition No. 1 and No. 2, respectively.

\section{and $300{ }^{\circ} \mathrm{C}$.}

Fig. 2 shows the photos, SEM image, and Raman spectra of the substrates after CVD with the condition No. 1 and No. 2. For $T_{\text {sub }}=500{ }^{\circ} \mathrm{C}(\mathrm{CVD}$ condition No. 1$)$, the substrate surface was blackened as shown in Fig. 2(a). To the contrary, for $T_{\text {sub }}=300{ }^{\circ} \mathrm{C}(\mathrm{CVD}$ condition No. 2), the color of the substrate surface was not changed as shown in Fig. 2(b).
In the SEM image of the substrates for $T_{\text {sub }}=500{ }^{\circ} \mathrm{C}$ [Fig. 2(c)], fibrous materials with $\sim 10 \mathrm{~nm}$ in diameter could be observed on the zeolite powder with several hundred nanometers in size. Such fibrous materials could not be observed for the substrates with $T_{\text {sub }}=300{ }^{\circ} \mathrm{C}$.

Fig. 2(d), and (e) show the Raman spectra of the substrates with $T_{\text {sub }}=500{ }^{\circ} \mathrm{C}$ and $300{ }^{\circ} \mathrm{C}$, respectively. In Fig. $2(\mathrm{~d})$, the radial breathing mode $(\mathrm{RBM})$ peaks were ob- 
served at low-frequency region. And the sharp and branched $G$ band peaks were observed around $1590 \mathrm{~cm}^{-1}$. Also, the $D$ band was observed around $1350 \mathrm{~cm}^{-1}$. These Raman bands are indicative of the presence of SWCNTs on the substrate. No obvious Raman signals, which indicates the presence of the CNTs were observed for $T_{\text {sub }}=300{ }^{\circ} \mathrm{C}$ [Fig. 2(e)].

From the results shown in Fig. 2, we could not synthesize SWCNTs at $T_{\text {sub }}=300{ }^{\circ} \mathrm{C}$. But we could successfully synthesized the SWCNTs at $T_{\text {sub }}=500{ }^{\circ} \mathrm{C}$. Though the $D$ band intensity, which relates the disorder of the CNTs is relatively high. This means that $T_{\text {sub }}=500{ }^{\circ} \mathrm{C}$ is still lower that the optimum substrate temperature. This substrate temperature is lower than the commonly-used substrate temperature $\left(T_{\text {sub }}=700{ }^{\circ} \mathrm{C}\right)$ [13]. Nonetheless these results are enough to confirm that the developed CVD apparatus works well as a normal cold-wall thermal CVD system.

\subsection{CNT growth with laser irradiation}

We performed the CVD process with laser irradiation. The $T_{\text {sub }}$ was $300{ }^{\circ} \mathrm{C}$. The laser fluence per pulse $(F)$ was controlled to be $0.05,1.1,2.2$, and $3.3 \mathrm{~J} / \mathrm{cm}^{2}$ by changing the laser spot size.

Fig. 3 shows the photos of the substrates after CVD with laser irradiation. For the irradiation conditions of $F=$ 3.3, 2.2, and $1.1 \mathrm{~J} / \mathrm{cm}^{2}$ [Fig. 3(a)-(c)], ablation of the catalyst and the substrate occurred at the laser spot. And almost whole surface of the substrate was blackened. For the irradiation condition of $F=0.05 \mathrm{~J} / \mathrm{cm}^{2}$ [Fig. 3(d)], ablation of the catalyst and the substrate did not occurred. And surface of the substrate was blackened only in the laser spot.

Fig. 4 shows the SEM images of the substrates after CVD with laser irradiation. At the laser spot for the irradiation condition of $F=3.3 \mathrm{~J} / \mathrm{cm}^{2}$ [Fig. 4(a)], the hole was observed on the substrate surface. There were any catalysts near the hole. The catalysts were also ablated by the laser irradiation. At the outside of the laser spot for the irradiation condition of $F=3.3 \mathrm{~J} / \mathrm{cm}^{2}$ [Fig. 4(b)], fibrous materials with $\sim 10 \mathrm{~nm}$ in diameter could be observed on the zeolite powder same as the Fig. 2(c). For the irradiation conditions of $F=2.2$, and $1.1 \mathrm{~J} / \mathrm{cm}^{2}$, similar SEM images were observed at the laser spot as Fig. 4(a). However, fibrous materials could not be observed at the outside of the laser spot. Fig. 4(c) shows the SEM image at the laser spot of the substrate for $F=0.05 \mathrm{~J} / \mathrm{cm}^{2}$. The fibrous materials could not be observed, while the zeolite powder was observed.

Fig. 5 shows the Raman spectra of the substrates for $F$ $=3.3,2.2,1.1$, and $0.05 \mathrm{~J} / \mathrm{cm}^{2}$. For the irradiation condition of $F=3.3 \mathrm{~J} / \mathrm{cm}^{2}$ [Fig. 5(a)], the Raman signals, which indicates the presence of the SWCNTs on the substrate, were observed at the outside of the laser spot, while the Raman signal from the substrate was observed at the laser spot. For the irradiation conditions of $F=2.2$, and $1.1 \mathrm{~J} / \mathrm{cm}^{2}[$ Fig. 5(b), (c)], the Raman spectra showed the similar trends each other. The Raman signals from the substrate were observed at the laser spots. Only the $D$ and $G$ bands were observed at the outside of the laser spot. The RBM signals were not observed at the outside of the laser spot. This result indicates that there are some carbonous materials at the outside of the laser spot on the substrates, but the materials don't have tubular structure. For the irradiation condition of $F=0.05 \mathrm{~J} / \mathrm{cm}^{2}$ [Fig. 5(d)], the Raman signals, which indicates the presence of the SWCNTs on the substrate, were observed at the laser spot. However, the intensities of RBM peaks and $G$ band were very small. This means that the number of synthesized SWCNTs is very small. We could not observe any fibrous materials in the SEM images as shown in Fig. 4(c). This might be due to that the SWCNTs were grown inside the zeolite powder.

The SEM images and Raman spectra, as shown in Fig. 4, and Fig. 5, show that the growth area and growth amount of CNTs depended on the laser fluence. In particular, for the laser fluence higher than $1.1 \mathrm{~J} / \mathrm{cm}^{2}$, CNTs were not synthesized at the laser spot, because the catalysts were ablated.
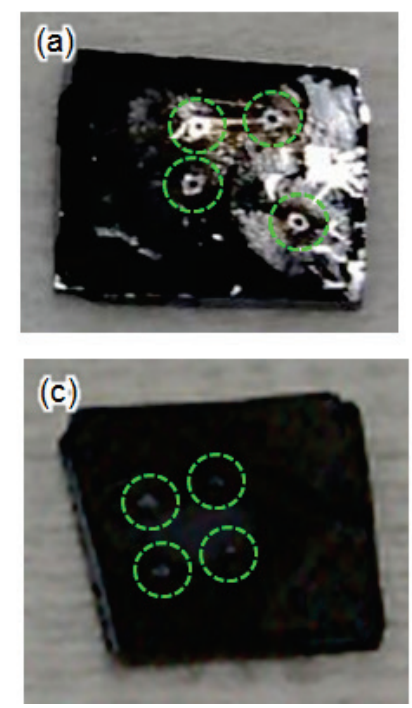
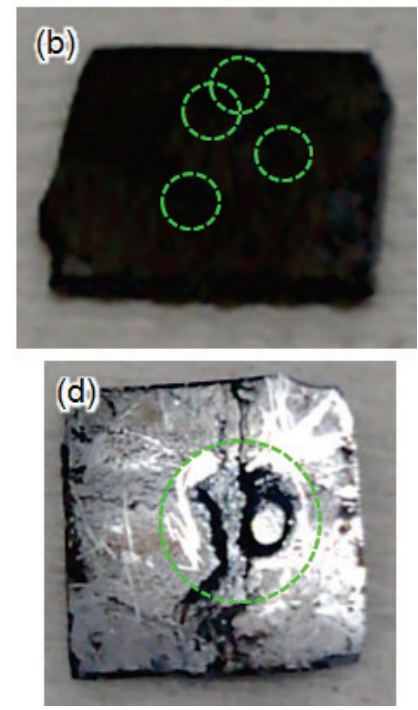

Fig. 3 Photos of the substrates after CVD with the condition (a) No. 3, (b) No. 4, (c) No. 5, and (d) No. 6. Green circles show the laser-irradiated area.
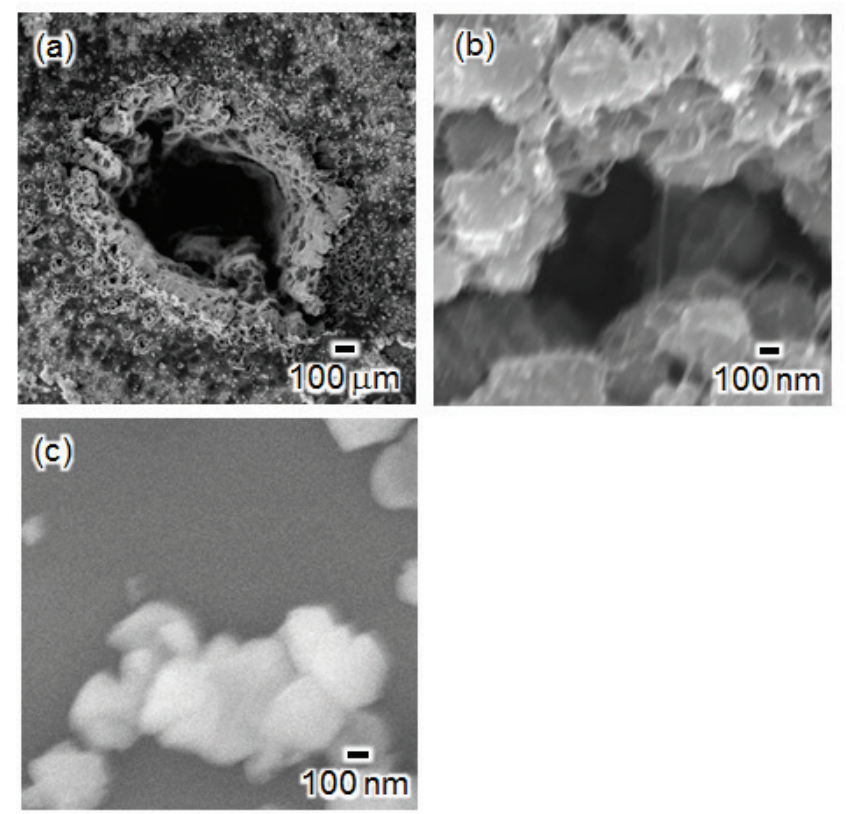

Fig. 4 SEM images of the substrates after CVD (a) with the condition No. 3 observed at the laser spot, (b) with the condition No. 3 observed at the outside of the laser spot, (c) with the condition No. 6 observed at the laser spot. 
(a)

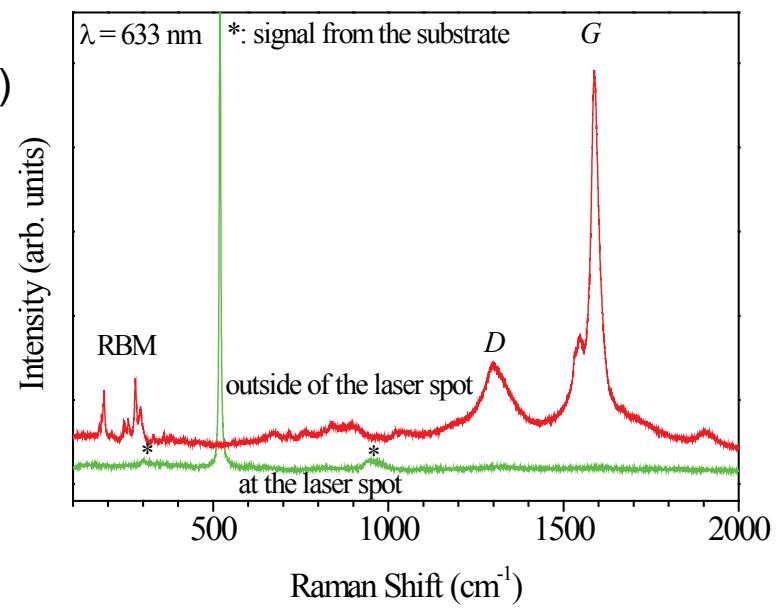

(c)

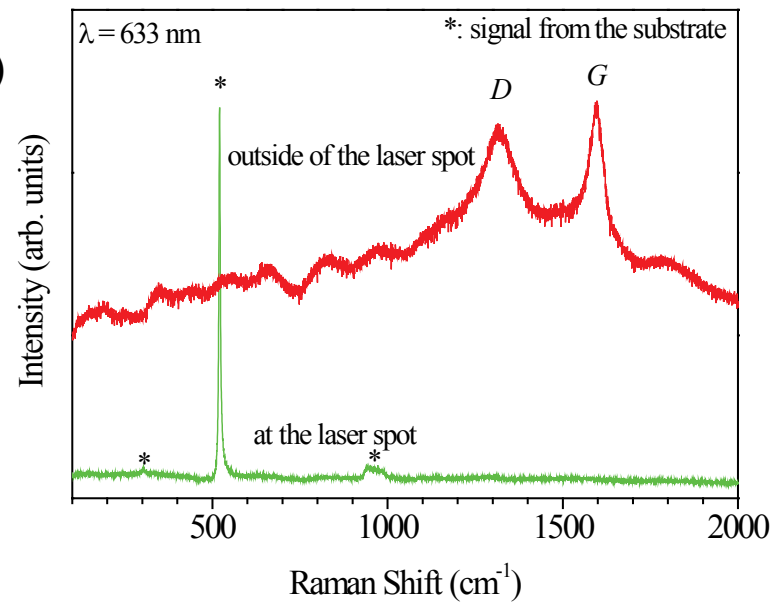

(b)

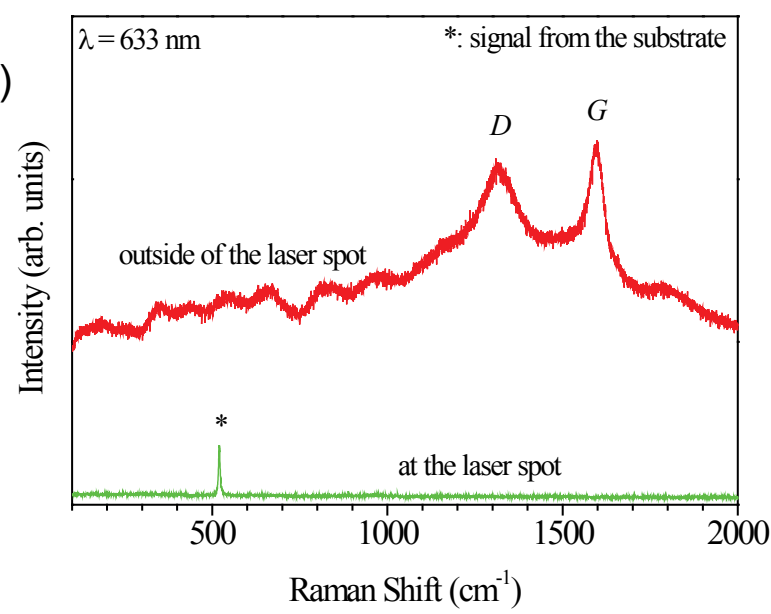

(d)

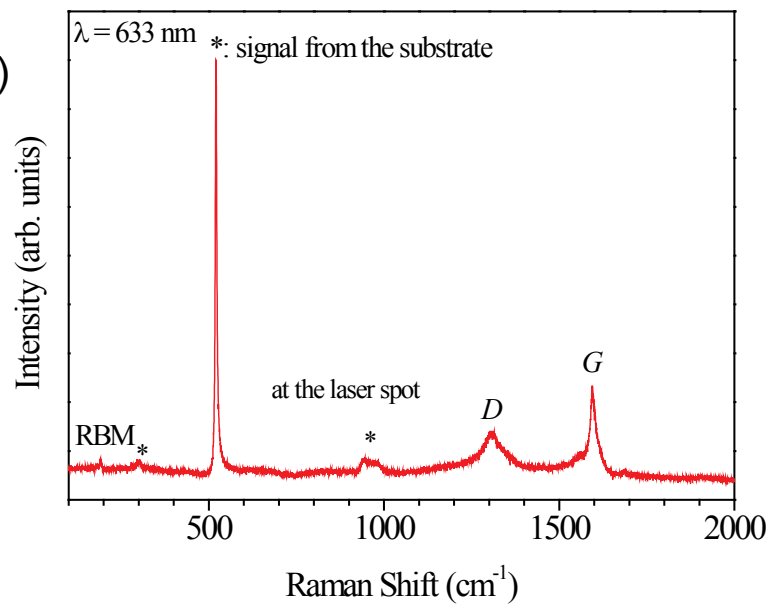

Fig. 5 Raman spectra of the substrates after CVD with the condition (a) No. 3, (b) No. 4, (c) No. 5, and (d) No. 6.

However the carbonous materials, including CNTs, were synthesized around the laser spot. This is due to the heating of the substrates and/or the dissociation of the carbon feedstock by laser irradiation. Actually, obvious change in the substrate temperature, which was measured by the thermocouple, was not observed. In the cases of $F=1.1$ and 2.2 $\mathrm{J} / \mathrm{cm}^{2}$, there are the effects of laser irradiation; the heating of the substrates and/or the dissociation of the carbon feedstock. But the effectiveness level of the laser irradiation was less for $F=1.1$ and $2.2 \mathrm{~J} / \mathrm{cm}^{2}$ than $F=3.3 \mathrm{~J} / \mathrm{cm}^{2}$. In other words, the heating of the substrates and the dissociation of the carbon feedstock were less for $F=1.1$ and 2.2 $\mathrm{J} / \mathrm{cm}^{2}$ than $F=3.3 \mathrm{~J} / \mathrm{cm}^{2}$. Therefore, the SWCNTs could not be synthesized for $F=1.1$ and $2.2 \mathrm{~J} / \mathrm{cm}^{2}$. For the laser fluence $0.05 \mathrm{~J} / \mathrm{cm}^{2}$, SWCNTs were synthesized at the laser spot on the substrate. In this case, we did not use any lens. The laser was a Gaussian beam. The flat-top beam is expected to be more suitable for the homogeneous growth of the CNTs in the irradiation spot.

\section{Summary}

We developed the LACVD system. We examined the operation of the developed CVD system without laser irradiation. After that, we performed the CVD process with laser irradiation. We investigated the dependence of the laser fluence on the grown CNTs. The growth area and growth amount of CNTs depends on the laser fluence. For the laser fluence $3.3 \mathrm{~J} / \mathrm{cm}^{2}$, the SWCNTs were synthesized at the outside of the laser spot. For the laser fluence 0.05 $\mathrm{J} / \mathrm{cm}^{2}$, SWCNTs were synthesized at the laser spot.

In the future, we will investigate the effect of the irradiation laser fluence further and the effect of the laser beam profile on the homogeneity of the grown SWCNTs. Also we will investigate the growth process of SWCNTs.

\section{Acknowledgments}

Part of this study has been supported by a grant from the INOUE ENRYO Memorial Foundation for Promoting Sciences organized by Toyo University in 2009, 2010, and by a Grant for the HighTech Research Centres organized by the Ministry of Education, Culture, Sports, Science and Technology (MEXT), Japan, since 2006.

\section{References}

[1] A. Jorio, G. Dresselhaus, M.S. Dresselhaus, Carbon Nanotubes. Advanced Topics in the Synthesis, Structure, Properties and Applications, Springer-Verlag, 2008.

[2] R. Saito, Physical Properties of Carbon Nanotubes, World Scientific Publishing Company, 1998.

[3] F. Rohmund, R.E. Morjan, G. Ledoux, F. Huisken, R. Alexandrescu, Journal of Vacuum Science \& Technology B 20 (2002) 802-811.

[4] Y. Fujiwara, K. Maehashi, Y. Ohno, K. Inoue, K. Matsumoto, Japanese Journal of Applied Physics Part 1Regular Papers Short Notes \& Review Papers. 44 (2005) 1581-1584. 
[5] S.N. Bondi, W.J. Lackey, R.W. Johnson, X. Wang, Z.L. Wang, Carbon. 44 (2006) 1393-1403.

[6] J. Shi, Y.F. Lu, K.J. Yi, Y.S. Lin, S.H. Liou, J.B. Hou, X.W. Wang, Applied Physics Letters. 89 (2006) 3.

[7] Z. Chen, Y. Wei, C.X. Luo, K.L. Jiang, L. Zhang, Q.Q. Li, S.S. Fan, J.C. Gao, Applied Physics Letters. 90 (2007) 3. [8] S. Chiashi, M. Kohno, Y. Takata, S. Maruyama, in: W.P. Hess, P.R. Herman, D. Bauerle, H. Koinuma (Eds.), COLA'05: 8th International Conference on Laser Ablation, Iop Publishing Ltd, Bristol, 2007, pp. 155-158.

[9] Z. Liu, D.J. Styers-Barnett, A.A. Puretzky, C.M. Rouleau, D. Yuan, I.N. Ivanov, K. Xiao, J. Liu, D.B. Geohegan, Applied Physics a-Materials Science \& Processing. 93 (2008) 987-993.

[10]H. Iwato, K. Namiki, K. Tamiya, Y. Tanaka, K. Suzuki, Applied Surface Science. 255 (2009) 9655-9658.

[11]J.B. Park, M.S. Jeong, S.H. Jeong, Applied Surface Science. 255 (2009) 4526-4530.

[12]J.B. Park, S.H. Jeong, M.S. Jeong, S.C. Lim, I.H. Lee, Y.H. Lee, Nanotechnology. 20 (2009) 7.

[13] S. Maruyama, R. Kojima, Y. Miyauchi, S. Chiashi, M. Kohno, Chem. Phys. Lett. 360 (2002) 229.

(Received: June 06, 2011, Accepted: November 14, 2011) 